\title{
Multiple phase transitions and magnetoresistance of $\mathrm{HoFe}_{4} \mathrm{Ge}_{2}$
}

\author{
J. Liu ${ }^{\mathrm{a}, \mathrm{b},{ }^{*},}$ V. K. Pecharsky ${ }^{\mathrm{a}, \mathrm{b}}$, and K. A. Gschneidner, Jr. ${ }^{\mathrm{a}, \mathrm{b}}$ \\ ${ }^{a}$ The Ames Laboratory, U.S. Department of Energy, Iowa State University, Ames, Iowa \\ 50011-3020,USA \\ ${ }^{\mathrm{b}}$ Department of Materials Science and Engineering, Iowa State University, Ames, Iowa \\ 50011-2300, USA
}

A systematic study of the structural, magnetic, heat capacity, electrical resistivity and magnetoresistance properties of $\mathrm{HoFe}_{4} \mathrm{Ge}_{2}$ has been performed. The temperature dependencies of the magnetization and heat capacity show three magnetic transitions at $T_{N}=51 \mathrm{~K}, T_{f 1}=42 \mathrm{~K}$, and $T_{f 2}=15 \mathrm{~K}$. The high temperature transition is antiferromagnetic ordering and the two low temperature phase transitions are due to rearrangements of the magnetic structure. A kinetically arrested phase is observed below a freezing point of $\sim 11 \mathrm{~K}$. Below $35 \mathrm{~K}$, the behavior of the isothermal magnetization reflects a first-order metamagnetic phase transition. Multiple phase transitions are also manifested in the electrical resistivity behavior. For a field change of $30 \mathrm{kOe}$, a large magnetoresistance of $\sim 30 \%$ is observed near $T_{f 2}(15 \mathrm{~K})$.

Keywords: Rare earth intermetallics, Magnetic properties, Heat capacity, Electrical resistivity, Magnetoresistance

\footnotetext{
* Corresponding author. E-mail address: liujing@iastate.edu (J. Liu)
} 


\section{Introduction}

Rare earth intermetallic compounds are of particular interest and importance in both fundamental research and various applications based on physical properties related to partially filled $4 f$ shells. For example, heavy fermion behavior [1], Kondo effect [2], quantum criticality [3], magnetocaloric effect [4], magnetoresistance [5] and magnetostriction [6] have been reported to occur in rare earth intermetallics. Therefore, investigation of lanthanide containing compounds is an important pathway towards discovery of new and interesting physical properties. Nearly 25 years ago, rare-earth ternary intermetallic compounds with general composition $\mathrm{R}: \mathrm{Fe}: \mathrm{Ge}=1: 4: 2(\mathrm{R}=\mathrm{Y}, \mathrm{Ho}, \mathrm{Dy}$, Er and $\mathrm{Lu}$ ) have been discovered and reported to crystallize with the tetragonal $\mathrm{ZrFe}_{4} \mathrm{Si}_{2}$ type structure at room temperature [7]. Low temperature neutron and x-ray diffraction investigations of $\mathrm{ErFe}_{4} \mathrm{Ge}_{2}, \mathrm{DyFe}_{4} \mathrm{Ge}_{2}$ and $\mathrm{HoFe}_{4} \mathrm{Ge}_{2}[8,9,10,11,12,13]$, reveal that these compounds order magnetically at low temperature and are paramagnetic at room temperature despite a relatively large concentration of Fe (57 at.\%). A study employing

${ }^{57} \mathrm{Fe}$ Mössbauer spectroscopy and magnetic measurements in high magnetic fields $[14,15]$ also confirmed the paramagnetic state at room temperature of these $\mathrm{RFe}_{4} \mathrm{Ge}_{2}$ intermetallics.

Among $\mathrm{RFe}_{4} \mathrm{Ge}_{2}$ compounds, $\mathrm{DyFe}_{4} \mathrm{Ge}_{2}[16]$ and $\mathrm{ErFe}_{4} \mathrm{Ge}_{2}$ [17] have been systematically investigated by magnetic measurements; both compounds exhibit interesting and complex magnetic behaviors. Both $\mathrm{DyFe}_{4} \mathrm{Ge}_{2}$ and $\mathrm{ErFe}_{4} \mathrm{Ge}_{2}$ were found to show three magnetic phase transitions, with two of them being associated with the magnetic structure changes and one being an antiferromagnetic (AFM) - paramagnetic (PM) transition. 
Besides, a reentrant magnetic glassy state was observed in $\mathrm{DyFe}_{4} \mathrm{Ge}_{2}$ below the freezing point of $15 \mathrm{~K}$, which is related to the geometrical frustration in the Fe sublattice, making the low temperature antiferromagnetic state metastable [16].

For the title compound $\mathrm{HoFe}_{4} \mathrm{Ge}_{2}$, x-ray and neutron diffraction experiments had been performed and reported in the past $[11,13]$ yet a detailed investigation of the physical properties, including magnetic, thermal and electrical, has not been carried out. Based on the results of the neutron diffraction study, $\mathrm{HoFe}_{4} \mathrm{Ge}_{2}$ exhibits three magnetic transitions, two of them being coupled magneto-elastic transitions at $T_{N}=52 \mathrm{~K}$ (from tetragonal $\mathrm{P}_{2} / \mathrm{mnm}$ paramagnetic to orthorhombic $\mathrm{Cmmm}$ antiferromagnetic) and $T_{i c l}=15 \mathrm{~K}$ (from orthorhombic $\mathrm{Cmmm}$ back to tetragonal $\mathrm{P} 4_{2} / \mathrm{mnm}$ ), the third being a purely magnetic structure change at $T_{c 2}=40 \mathrm{~K}$. The peculiar re-entrant magneto-elastic transition at $T_{i c l}$ of $\mathrm{HoFe}_{4} \mathrm{Ge}_{2}$ at low temperature is unique among other $\mathrm{RFe}_{4} \mathrm{Ge}_{2}$ compounds. This interesting behavior of $\mathrm{HoFe}_{4} \mathrm{Ge}_{2}$ warrants further investigation of its low temperature magnetic state to uncover the nature of the complex behavior. It is also of interest to study its magnetocaloric and magnetoresistance behaviors. Therefore, in this paper, we present a detailed investigation of the low temperature antiferromagnetic state by using static and dynamic magnetic measurements coupled with heat capacity, and electrical resistivity, and magnetoresistance measurements of $\mathrm{HoFe}_{4} \mathrm{Ge}_{2}$.

\section{EXPERIMENTAL DETAILS}

The $\mathrm{Ho}$ and $\mathrm{Fe}$ metals used to prepare the $\mathrm{HoFe}_{4} \mathrm{Ge}_{2}$ compound were obtained from the Materials Preparation Center of the Ames Laboratory [18] and were 99.8+ at. \% and 
99.98 wt. \% pure respectively with respect to all other elements in the Periodic Table including the interstitial impurity elements $\mathrm{H}, \mathrm{C}, \mathrm{O}$ and N. The Ge with a 99.999 wt. \% purity was purchased from Alfa Aeser, Inc. A polycrystalline button of $\mathrm{HoFe}_{4} \mathrm{Ge}_{2}$ was prepared by arc melting of the stoichiometric mixture of constituent elements under an argon atmosphere. The alloy was flipped and re-melted four times to ensure the compositional homogeneity. The crystal structure and phase purity of the sample were studied by X-ray powder diffraction (XRD) using PANalytical X'Pert diffractometer with $\mathrm{Cu} \mathrm{K} \alpha_{1}$ radiation at room temperature. Rietica LHPM software [19] was employed to perform a Rietveld refinement. The alloy adopts the $\mathrm{ZrFe}_{4} \mathrm{Si}_{2}$-type tetragonal structure with the lattice parameters $a=7.2863$ (5) $\AA, c=3.8685(3) \AA$ at room temperature. Minor amounts of $\mathrm{Fe}\left(\sim 5\right.$ wt. \%) and $\mathrm{HoFe}_{2} \mathrm{Ge}_{2}(\sim 4.5$ wt. \%) have been detected as impurity phases. The ac and dc magnetic measurements were carried out using a superconducting quantum interference device (SQUID) magnetometer MPMS XL-7. Isothermal magnetization up to $140 \mathrm{kOe}$ magnetic field was measured using Physical Property Measurement System (PPMS) from Quantum Design Inc. Heat capacity data were collected on heating in an automatic semi-adiabatic heat pulse calorimeter [20] in the temperature range between 2 and $350 \mathrm{~K}$ at various magnetic fields after cooling the sample in a zero magnetic field from room temperature.

The sample for the electrical resistivity measurements with dimensions $9.3 \times 1.2 \times 1.5$ $\mathrm{mm}^{3}$ was cut from the polycrystalline button. The electrical resistivity was measured using a standard four-probe method. The temperature $(T)$ and magnetic field $(H)$ dependencies of the electrical resistivity $(\rho)$ were measured with a constant ac excitation 
electrical current of $25 \mathrm{~mA}$ and frequency $35 \mathrm{~Hz}$ in the temperature range between 2 and $300 \mathrm{~K}$ and in magnetic fields between 0 and $70 \mathrm{kOe}$. These experiments were performed using PPMS apparatus. The temperature dependencies of the electrical resistivity, $\rho(T)$ were measured in the zero-field-cooled heating (ZFC) and field-cooled cooling (FCC) modes. The isothermal $\rho(H)$ measurements were made after thermal demagnetization by heating to $150 \mathrm{~K}$ and then cooling down to the measurement temperature in a zero magnetic field. The magnetoresistance ratio was calculated as $M R=\left(\rho_{H}-\rho_{0}\right) / \rho_{0} \times 100 \%$, where $\rho_{H}$ and $\rho_{0}$ is the electrical resistivity measured in a magnetic field $H$ and zero magnetic field, respectively.

\section{Results and discussion}

\subsection{Magnetic properties}

Figure 1 shows the dc magnetization of $\mathrm{HoFe}_{4} \mathrm{Ge}_{2}$ measured as a function of temperature in a magnetic field of $1 \mathrm{kOe}$. In order to clearly depict the phase transitions, the figure only shows the magnetization over the temperature range from 2 to $100 \mathrm{~K}$. The zerofield-cooled heating (ZFC) magnetization shows five anomalies: a kink at $51 \mathrm{~K}\left(T_{N}\right)$, a cusp at $42 \mathrm{~K}\left(T_{f 1}\right)$ (also see inset in Fig. 1), a sharp peak at $15 \mathrm{~K}\left(T_{f 2}\right)$, a deep minimum at $11 \mathrm{~K}\left(T_{f 3}\right)$ and a small peak at $4 \mathrm{~K}\left(T_{f 4}\right)$. In agreement with the neutron diffraction measurements of $\mathrm{HoFe}_{4} \mathrm{Ge}_{2}$ at low temperature [13], the transition at $51 \mathrm{~K}$ reflect bulk antiferromagnetic ordering. The low temperature XRD analysis $[5,11]$ also indicated that this antiferromagnetic transition is coupled with a structural transition from the tetragonal $\left(P 4_{2} / m n m\right.$ symmetry) to a distorted orthorhombic structure that adopts $C m m m$ symmetry. 
Transitions at $T_{f 1}$ and $T_{f 2}$, are both related to a magnetic structure change within the antiferromagnetic state. However, the transition at $15 \mathrm{~K}\left(\mathrm{~T}_{f 2}\right)$ is also related with a structural transition from the orthorhombic back to the original tetragonal (space group $\left.P 4_{2} / m n m\right)$ structure [11]. With regard to the anomalies at $T_{f 3}$ and $T_{f 4}$, nothing was observed in either the low temperature XRD or neutron diffraction measurements. Is it known that $\mathrm{HoFe}_{2} \mathrm{Ge}_{2}$ exhibits a long range antiferromagnetic ordering below $1.6 \mathrm{~K}$ being paramagnetic above this temperature [21], and therefore, two minor impurity phases do not play a role in these magnetic anomalies. Further, $T_{f 3}$ is $9 \mathrm{~K}$ in the FCC protocol and the small peak at $T_{f 4}$ can only be observed in the ZFC curve. A similar phenomenon was also found in $\mathrm{DyFe}_{4} \mathrm{Ge}_{2}$ and explained as a freezing point signaling the reentrant glassy state. ${ }^{16}$ Therefore, in the case of $\mathrm{HoFe}_{4} \mathrm{Ge}_{2}$, a metastable state may also form in the low temperature regime due to geometrical frustration arising from the antiferromagnetic Fe-Fe interactions, as discussed in Ref. [16].

Below $15 \mathrm{~K}$, a strong thermomagnetic irreversibility is observed between the field-cooled cooling (FCC) and field-cooled warming (FCW) plots. A noticeable thermal hysteresis of $2 \mathrm{~K}$ between FCW and FCC curves at $T_{f 2}$ suggests that this is a first-order phase transition [22], confirming the XRD results of Ref. 11. It is worth noting that this structural phase transition is incomplete during cooling based on the analysis of XRD results [11]. From the inset of Fig. 1, the expanded range near the $T_{f 1}$ transition temperature, a small irreversibility between the FCW and FCC is also observed and from the low temperature XRD results [11], this transition is also first order. 
Temperature dependent magnetization of $\mathrm{HoFe}_{4} \mathrm{Ge}_{2}$ was also measured under applied fields of 5, 10, 15, 25, 30 and $50 \mathrm{kOe}$, see Fig. 2. Magnetic phase transitions at $T_{N}, T_{f 1}, T_{f 2}$ and $T_{f 3}$ can still be clearly observed at $H=5,10$ and $15 \mathrm{kOe}$. The anomaly at the lowest temperature $\sim 4 \mathrm{~K}$ is not observed in fields higher than $1 \mathrm{kOe}$ and the peak at $T_{f 2}$ in $H=$ $15 \mathrm{kOe}$ is smoothed out compared to that at $H=1 \mathrm{kOe}$ (Fig. 1). The temperature of the transition at $T_{f 2}$ decreases from $15 \mathrm{~K}$ in $1 \mathrm{kOe}$ to $11 \mathrm{~K}$ in $25 \mathrm{kOe}$. Below $H=15 \mathrm{kOe}$, substantial thermomagnetic irreversibility among ZFC, FCC, and FCW curves below $T_{f 2}$ remains.

The location of the peak at $T_{f l}$ (ZFC data) decreases from $42 \mathrm{~K}$ with $H=1 \mathrm{kOe}$ to $39 \mathrm{~K}$ with $H=15$ kOe. The thermomagnetic irreversibility between FCW and FCC curves associated with $T_{f 1}$ becomes more obvious with the increasing applied field and is most evident when $H$ is $15 \mathrm{kOe}$, where $M_{\mathrm{FCC}}$ is higher than $M_{\mathrm{FCW}}$ from 39 to $28 \mathrm{~K}$ (see inset in Fig. (2c). Below $H=15 \mathrm{kOe}$, the transition at $T_{f 1}$ exhibits thermal hysteresis of $1 \mathrm{~K}$ between the heating and warming curves. With a further increase of the magnetic field, the transition at $T_{f 1}$ shifts downward to $34 \mathrm{~K}$, smears out, and $M(T)$ displays only a small thermal irreversibility at $H=25 \mathrm{kOe}$; it finally disappears when magnetic field reaches and exceeds $30 \mathrm{kOe}$.

In 30 and 50 kOe magnetic fields, a bifurcation between ZFC and FCC is clearly observed starting from 14 and $12 \mathrm{~K}$, respectively. Apart from the bifurcation, the ZFC curves of $30 \mathrm{kOe}$ magnetic fields also show a small peak around $8 \mathrm{~K}$, which is related to the transition at $T_{f 2}$. The inset of Fig. 2(e) shows the field dependence of the temperature 
of the peak at $T_{f 2}$. Further, it appears that the anomaly at $T_{N}$ may become a ferrimagnetic to paramagnetic transition at $H=30$ and $50 \mathrm{kOe}$ due to the high magnetic fields which can change the magnetic state of the compound from antiferromagnetic to ferrimagnetic.

To further ascertain the magnetic states of $\mathrm{HoFe}_{4} \mathrm{Ge}_{2}$, Fig. 3(a) shows the real ( $\left.\chi^{\prime}\right)$ component and Fig. 3(b) shows the imaginary ( $\chi$ ”) component of the ac magnetic susceptibility as a function of temperature measured in zero dc magnetic fields. The measurements were performed on heating in a 5 Oe ac field at frequencies of 1, 10, 100, and $1000 \mathrm{~Hz}$. The magnetic anomalies associated with the first order phase transition at $T_{f 2}=15 \mathrm{~K}$ and magnetic ordering at $T_{N}=51 \mathrm{~K}$ are clearly seen as a sharp peak and a broad cusp, respectively, in $\chi^{\prime}(\mathrm{T})$ plots. The anomaly associated with the magnetic transition at $T_{f 1}=42 \mathrm{~K}$ is seen as a slope change at $\sim 40 \mathrm{~K}$. A small shoulder at about 11 $\mathrm{K}\left(T_{f 3}\right)$ is observed at $1 \mathrm{~Hz}$ applied frequency and it diminishes with increasing frequency. The small peak at $4 \mathrm{~K}$ observed in the $1 \mathrm{kOe}$ applied filed $M(T)$ data is not observed in the $\chi^{\prime}(T)$ measurements. Moreover, $\chi^{\prime}(T)$ is practically independent of the frequency above $T_{f 2}$, exhibiting weak but clear frequency dependence at and below $T_{f 2}$. The behavior of the imaginary component clearly reflects phase transitions at $T_{f 2}$ and $T_{f 3}$. Transition at $T_{N}$ is not seen due to the absence of magnetic domains in the AFM structure. $T_{f 1}$ is identified as the increased energy loss process that starts around $40 \mathrm{~K}$. We note that the nearly constant positive background observed above $\sim 40 \mathrm{~K}$ in 1,10 and $100 \mathrm{~Hz}$ data is due to the presence of a minor amount of ferromagnetic iron impurity in the sample. Its presence is likely responsible for the unphysical negative and highly scattered values of $\chi$ ” observed in $1000 \mathrm{~Hz}$ data. 
Figure 4 shows the isothermal magnetization of $\mathrm{HoFe}_{4} \mathrm{Ge}_{2}$ measured from $5 \mathrm{~K}$ to $60 \mathrm{~K}$. Each curve was obtained by zero field cooling from the paramagnetic state to the desired temperature. A weak ferromagnetic signature is seen in all $M(H)$ data because of a small amount ( 5 wt.\%) of the ferromagnetic Fe impurity. In fields less than $140 \mathrm{kOe}$, the magnetization only shows one metamagnetic phase transition, which is different from $\mathrm{DyFe}_{4} \mathrm{Ge}_{2}$ which displays two metamagnetic phase transitions [16]. The magnetization decreases with increasing temperature. The metamagnetic phase transition of $\mathrm{HoFe}_{4} \mathrm{Ge}_{2}$ is observed up to $35 \mathrm{~K}$ and accompanied with measurable magnetic hysteresis, indicating the first-order character of the metamagnetic process. Moreover, the hysteresis decreases as the temperature increases and disappears at $\sim 35 \mathrm{~K}$. The critical field $(H c)$ of the metamagnetic transition is defined as the maximum of the derivative of magnetization with respect to the applied magnetic field. It is found that $H c$, as shown in Fig. 4(c), is 22 kOe at 5 and $10 \mathrm{~K}$, shifts to $24 \mathrm{kOe}$ at 15 and $20 \mathrm{~K}$, and then decreases with increasing temperature. In addition, comparing the critical fields in the magnetization and demagnetization process, there are about $\sim 2$ kOe hysteresis associated with the metamagnetic transition.

The isothermal magnetization curves at 2, 5, 10 and $15 \mathrm{~K}$ are cycled twice, as shown in Fig. 5, to further study the magnetic state of $\mathrm{HoFe}_{4} \mathrm{Ge}_{2}$ at low temperature. It is clear that at 2 and $5 \mathrm{~K}$, the second-field-increase magnetization curves lie between the virgin magnetization and demagnetization curves, indicating that the metamagnetic transition is only partially reversible in this temperature range. When temperature is increased to 10 
$\mathrm{K}$, the second magnetization curve becomes identical to the first magnetization curve, showing that the metamagnetic transition becomes completely reversible. This behavior is similar to that observed in $\mathrm{DyFe}_{4} \mathrm{Ge}_{2}$ [16], and we believe that it indicates the presence of a low temperature region, where a system becomes kinetically arrested, i.e. a freezing point occurs in $\mathrm{HoFe}_{4} \mathrm{Ge}_{2}$ below $T_{f 3}(\sim 9 \mathrm{~K}$ during zero-field cooling process). Hence, in addition to a conventional metamagnetic phase transition boundary in temperaturemagnetic field coordinates, there is an additional boundary defining a freezing/unfreezing, glass-like transition. This freezing boundary overlaps with the metamagnetic transition boundary and a part of the material becomes kinetically arrested.

In order to further study the low temperature magnetic glassy state of $\mathrm{HoFe}_{4} \mathrm{Ge}_{2}$, we carried out relaxation measurements and the results are shown in Fig. 6. The measurements were carried out after zero field cooling the sample from paramagnetic state to the desired temperature. After reaching the temperature, a field of $1 \mathrm{kOe}$ was applied for $1000 \mathrm{~s}$ and the time dependence of magnetization was measured immediately after the field was switched off. The isothermal remanent magnetization shows strong time dependence and there is no saturation even after $\sim 4$ hours $(15,000 \mathrm{sec})$. The observation of such slow relaxation confirms the presence of a metastable phase due to kinetic arrest noted above. The time decay of the remanent magnetization can be well described by a superposition of a stretched exponential and a constant term, $M=M_{0}+$ $M_{1} \exp \left[-(t / \tau)^{1-n}\right]$, where $M_{0}, M_{1}$ are the fitting parameters, $\tau$ is the relaxation time constant, and $n$ is the stretching parameter [23]. The best fit to this expression gives $M_{0}=0.9595$ $\mathrm{emu} / \mathrm{g}, M_{1}=0.04073 \mathrm{emu} / \mathrm{g}, \tau=1084 \mathrm{~s}$ and $n=0.46$. 


\subsection{Heat capacity}

Temperature dependencies of heat capacity $\left(C_{P}\right)$ of $\mathrm{HoFe}_{4} \mathrm{Ge}_{2}$ were measured in $0,1,10$, 30 and 50 kOe magnetic fields, as shown in Fig. 7. The zero field heat capacity data presents two clear anomalies around 14 and $50 \mathrm{~K}$, which are close to $T_{f 2}$ and $T_{N}$, respectively. At $T_{N}$, the anomaly is quite broad and it weakly dependent on the applied field. The transition at $T_{f 2}$ is a minor $\lambda$-like anomaly; with increasing magnetic field, the $C_{P}(T)$ peak decreases and shifts toward lower temperature. Even though the shape of the anomaly at $T_{f 2}$ is inconsistent with the first order nature of this transition, the existence of the low temperature tail that makes it look $\lambda$-like is related to the fact that the tetragonal to orthorhombic transformation on heating is sluggish, which is consistent with it being incomplete on cooling. At $50 \mathrm{kOe}$, the peak associated with $T_{f 2}$ disappears (Fig. 7a inset), indicating that the high magnetic field suppresses the first order transition at $T_{f 2}$. The transition at $T_{f 1}=42 \mathrm{~K}$ is only observed in heat capacity data as a minor anomaly around $40 \mathrm{~K}$ in magnetic fields below $30 \mathrm{kOe}$ (inset b of Fig. 7). This anomaly is also unusual considering the first order nature of this transition as based on the $M(T)$ and the neutron diffraction data [13]. All of these results confirms that the magnetic ordering in $\mathrm{HoFe}_{4} \mathrm{Ge}_{2}$ is quite complex. Moreover, the magnetocaloric effect for the three phase transitions $\left(T_{f 1}, T_{f 2}\right.$ and $\left.T_{N}\right)$, was estimated using both the $M(H)$ data and $C_{P}(T)$ data and is small $\left(\Delta S_{\mathrm{M}} \leq-1.5 \mathrm{~J} / \mathrm{kg} \mathrm{K}\right)$. 
The $M(H), M(T)$ and $C_{P}(T)$ behaviors of $\mathrm{HoFe}_{4} \mathrm{Ge}_{2}$ at low temperature discussed above (i.e., strong irreversibility between FCW and FCC data, the FCW curves lie below the FCC ones, the temperature dependence of the reversibility of the field-induced metamagnetic phase transition, the weak $\chi^{\prime}(T)$ frequency dependence below $T_{f 2}$, the long-time relaxation of the isothermal remanent magnetization and no clear anomalies at $T_{f 3}$ and $T_{f 4}$ in $C_{P}(T)$ data) suggest the presence of a metastable phase which is partially kinetically arrested below the freezing point $(\sim 9 \mathrm{~K})$. A kinetically arrested metastable phase has also been observed in $\mathrm{DyFe}_{4} \mathrm{Ge}_{2},{ }^{16}$ and is much more pronounced in $\mathrm{Gd}_{5} \mathrm{Ge}_{4}$ [24].

\subsection{Electrical resistivity}

Normally, electrical resistivity behavior can also reflect a magnetic state change. Therefore, to further study the multiple magnetic phase transitions in $\mathrm{HoFe}_{4} \mathrm{Ge}_{2}$, the temperature dependencies of the electrical resistivity of $\mathrm{HoFe}_{4} \mathrm{Ge}_{2}$ upon heating and cooling in $0,1,10,20$ and $30 \mathrm{kOe}$ magnetic fields were measured and are displayed in Fig. 8. The measurement on heating was made after the sample was cooled in a zero magnetic field. The result demonstrates the metallic character of this compound. The curves measured on heating and cooling in zero magnetic field coincide above $\sim 15 \mathrm{~K}$. Under zero field, the electrical resistivity exhibits a well-defined peak at about $11 \mathrm{~K}$ (ZFC) and $8 \mathrm{~K}$ (FCC) and a sharp minimum at $15 \mathrm{~K}$ (ZFC) and $12 \mathrm{~K}$ (FCC). Thermal irreversibility between ZFC and FCC data is observed from 7 to $15 \mathrm{~K}$. From the first derivative of the electrical resistivity with respect to the temperature $d \rho / d T$, three clear anomalies are observed near $T_{N}=51 \mathrm{~K}, T_{f 1}=39 \mathrm{~K}$ and $T_{f 2}=13 \mathrm{~K}$. At $T_{N}$, the temperature 
dependence of $d \rho / d T$ changes abruptly and is due to the onset of the antiferromagnetic ordering. These three anomalies in the resistivity data are in accord with the magnetic measurements and heat capacity data, indicating that these three magnetic phase transitions are dominant. A linear temperature dependence of the resistivity is observed in the antiferromagnetic state between $T_{f 2}$ and $T_{f 1}$ as well as in the paramagnetic state. But the slope of the electrical resistivity is larger below the Néel temperature because of the reduced spin-disorder scattering brought about by the long range antiferromagnetic ordering. Linearity of electrical resistivity in the paramagnetic state suggests the presence of dominant phonon contribution to the electrical resistivity [25].

Comparing the electrical resistivity behaviors under various applied fields, we note that for high magnetic fields, e.g. at $H=30 \mathrm{kOe}$ the low temperature peak disappears in both ZFC and FCC curves and at $H=25 \mathrm{kOe}$, only ZFC manifests the peak at $8 \mathrm{~K}$. Under a $10 \mathrm{kOe}$ magnetic field, the peak occurs at 10 and $8 \mathrm{~K}$ in $\mathrm{ZFC}$ and FCC curves, respectively. In addition, under the fields of 10 and $20 \mathrm{kOe}$, the thermal irreversibility between the heating and cooling curves is observed from the electrical resistivity minima (14 K) down to $2 \mathrm{~K}$.

Figure 9(a) shows the temperature dependence of the magnetoresistance (MR) at 30 kOe. A negative MR effect is observed below the $11 \mathrm{~K}$ (freezing point), then changes to positive at high temperature and achieves the maximum of $\sim 30 \%$ at $15 \mathrm{~K}\left(T_{f 2}\right)$. A $\sim 2 \mathrm{~K}$ hysteresis between the heating and cooling process is in agreement with the first-order nature at $T_{f 2}$. Figure $9(\mathrm{~b})$ depicts the magnetic field dependence of magnetoresistance at 
various temperatures from 2 to $50 \mathrm{~K}$ in $\mathrm{HoFe}_{4} \mathrm{Ge}_{2}$. The curves at and below $30 \mathrm{~K}$ show a metamagnetic transition indicated by a sharp increase in MR over a certain field range. A positive MR effect of about $30 \%$ is observed when $\mathrm{H}$ is greater than the critical field of $22 \mathrm{kOe}$ at $15 \mathrm{~K}$, which coincides with the first-order field-induced phase transition as observed in the magnetization data The demagnetization curves always lie above the magnetization curve as expected due the existence of the hysteresis effect which is exactly the same as the magnetization curves. We note that the magnitude of the magnetoresistance is comparable with that of other intermetallic compounds which have first-order metamagnetic transitions such as $\mathrm{Gd}_{5}\left(\mathrm{Si}_{1.8} \mathrm{Ge}_{2.2}\right)$ [26], $\mathrm{Ce}\left(\mathrm{Fe}_{0.93} \mathrm{Ru}_{0.07}\right)_{2}$ [27], $\mathrm{SmMn}_{2} \mathrm{Ge}_{2}$ [28]. It is interesting to note that while decreasing the field at $2 \mathrm{~K}$, MR crosses the initial curve (obtained during the increasing field) and becomes positive below $22 \mathrm{kOe}$ and remains constant at about $15 \%$ from $18 \mathrm{kOe}$ to zero. This behavior is consistent with the presence of the metastable, kinetically arrested phase when the sample is cooled in a zero magnetic field below the freezing point.

As discussed in the previous section, $\mathrm{HoFe}_{4} \mathrm{Ge}_{2}$ shows a metamagnetic transition around $22 \mathrm{kOe}$. The value of $H c$, defined from the peak point in the $\mathrm{d} \rho / \mathrm{dH}$ curves, exhibits almost no temperature dependence, which compares very well with that previously obtained from isothermal magnetization curves. A hysteresis of $\sim 2 \mathrm{kOe}$ has also been observed between the increasing and decreasing branches of the magnetic field, which is consistent with the hysteresis in the magnetic measurements. It is also worth mentioning that the MR is almost constant after the field-induced transition finished. When the temperature is equal and higher than $40 \mathrm{~K}$, where field-induced metamagnetic transition 
disappears, the MR is almost zero and independent of the field. Therefore, the MR effect in $\mathrm{HoFe}_{4} \mathrm{Ge}_{2}$ is strongly correlated with the field-induced magnetic phase transformation.

\section{Conclusions}

We have studied the thermal, magnetic, and electrical properties of $\mathrm{HoFe}_{4} \mathrm{Ge}_{2}$. The results reveal the existence of a complex spin reorientation process within the AFM phase at low temperatures of $\mathrm{HoFe}_{4} \mathrm{Ge}_{2}$. PM to AFM transition takes place at $T_{\mathrm{N}}=51 \mathrm{~K}$. Two additional first-order magnetic phase transitions take place at $T_{f 1}=42 \mathrm{~K}$ and $T_{f 2}=15 \mathrm{~K}$. A kinetically arrested state is observed below $\sim 10 \mathrm{~K}$ and the isothermal remanent magnetization undergoes slow relaxation with time at $5 \mathrm{~K}$. The low temperature physical properties manifest strong magnetic field dependencies. Both magnetization and electrical resistivity measurements as a function of applied filed provide evidences for first-order metamagnetic transition at critical field $\sim 22 \mathrm{kOe}$ with strong hysteresis in $\mathrm{HoFe}_{4} \mathrm{Ge}_{2}$. The interesting and unusual MR behavior - the negative $\Delta \rho / \rho$ at low temperatures and a change of sign at higher temperature - is observed. For a field change of $30 \mathrm{kOe}$, a large MR of $~ 30 \%$ is observed near $T_{f 2}$.

\section{ACKNOWLEDGMENTS}

This work was supported by the U. S. Department of Energy, Office of Basic Energy Science, Division of Materials Sciences and Engineering. The research was performed at the Ames Laboratory operated for the U. S. Department of Energy by Iowa State University under Contract No. DE-AC02-07CH11358. 


\section{References}

[1] T. Brugger, T. Schreiner, G. Roth, P. Adelmann, and G. Czjzek, Phys. Rev. Lett. 71, (1993) 2481.

[2] J. Stankiewicz, M. Evangelisti, Z. Fisk, P. Schlottmann, and Lev P. Gor'kov, Phys. Rev. Lett. 108, (2012) 257201.

[3] A. V. Chubukov, S. Sachdev, and J. Ye, Phys. Rev. B 49, (1994) 11919.

[4] V. K. Pecharsky, and K. A. Gschneidner, Jr., Phys. Rev. Lett. 78, (1997) 4494.

[5] E. M. Levin, V. K. Pecharsky, and K. A. Gschneidner, Jr., Phys. Rev. B 60, (1999) 7993.

[6] L. Morellon, P. A. Algarabel, M. R. Ibarra, J. Blasco, B. Garcia-Landa, Z. Arnold, and F. Albertini, Phys. Rev. B 58, (1998) R14721.

[7] O. Ya. Oleksyn, Yu. K. Gorelenko and O. I. Bodak, Proc. Tenth Int. Conf. Solid Compounds of Transition Elements, Munster, (1991).

[8] P. Schobinger-Papamantellos, J. Rodríguez-Carvajal, G. Andre, C. H. De Groot, F. R. De Boer, and K. H. J. Buschow, J. Magn. Magn. Mater. 191, (1999) 261.

[9] P. Schobinger-Papamantellos, J. Rodríguez-Carvajal, K. H. J. Buschow, E. Dooryhee and A. N. Fitch, J. Magn. Magn. Mater. 210, (2000) 121.

[10] P. Schobinger-Papamantellos, J. Rodríguez-Carvajal, K. H. J. Buschow, E. Dooryhee and A. N. Fitch, J. Magn. Magn. Mater. 300, (2006) 315.

[11] P. Schobinger-Papamantellos, J. Rodríguez-Carvajal, K. H. J. Buschow, E. Dooryhee and A. N. Fitch, J. Magn. Magn. Mater. 250, (2002) 225. 
[12] P. Schobinger-Papamantellos, J. Rodríguez-Carvajal, G. André, and K. H. J. Buschow, J. Magn. Magn. Mater. 300, (2006) 333.

[13] P. Schobinger-Papamantellos, J. Rodríguez-Carvajal, G. André, C.Ritter, and K. H. J. Buschow, J. Magn. Magn. Mater. 280, (2004) 119.

[14] A. M. Mulders, P. C. M Gubbens, Q. A. Li, F. R. de Boer and K. H. J. Buschow. J. Alloys Comp. 221, (1995) 197.

[15] P. C. M. Gubbens, B. D. van Dijk, A. M. Mulders, S. J. Harker and K. H. J. Buschow, J. Alloys Comp. 319, (1995) 1.

[16] J. Liu, D. Paudyal, Y. Mudryk, J. D. Zou, K. A. Gschneidner, Jr., and V. K. Pecharsky, Phys. Rev. B 88, (2013) 014423.

[17] F. Canepa , S. Cirafici, F. Merlo, M. Pani, L. Carpaneto, M. R. Cimberle, J. Alloys Comp. 266, (1998) 26.

[18] Materials Preparation Center, Ames Laboratory US-DOE, Ames, IA, USA, www.mpc.ameslab.gov

[19] B. Hunter, Rietica-A Visual Rietveld Program, International Union of Crystallography Commission on Powder Diffraction Newsletter No. 20 (1998), http://www.rietica.org.

[20] V. K. Pecharsky, J. O. Moorman, and K. A. Gschneidner, Jr., Rev. Sci. Instrum 68, (1997) 4196.

[21] S. Baran, A. Hoser, D. Kaczorowski, K. Kiefer, B. Penc, and A. Szytuła, Solid State Commun. 149, (2009) 1261. 
[22] S. B. Roy, G. K. Perkins, M. K. Chattopadhyay, A. K. Nigam, K. J. S. Sokhey, P. Chaddah, A. D. Caplin, and L. F. Cohen, Phys. Rev. Lett. 92, (2004) 147203.

[23] M. Gabay and G. Toulouse, Phys. Rev. Lett. 47, (1981) 201.

[24] S. B. Roy, M. K. Chattopadhyay, P. Chaddah, J. D. Moore, G. K. Perkins, L. F. Cohen, K. A. Gschneidner, Jr., and V. K. Pecharsky, Phys. Rev. B 74, (2006) 012403.

[25] R. Nirmala, V. Sankaranarayanana, K. Sethupathi, A.V. Morozkin, A. G. Joshi, S. K. Malik, J. Magn. Magn. Mater. 309, (2007) 212.

[26]L. Morellon, J. Stankiewicz, B. García-Landa, P. A. Algarabel, and M. R. Ibarra, Appl. Phys. Lett. 73, (1998) 3462.

[27] H. P. Kunkel, X. Z. Zhou, P. A. Stampe, J. A. Cowen and G. Williams, Phys. Rev. B 53, (1996) 15099.

[28] E. V. Sampathkumaran, P. L. Paulose, and R. Mallik, Phys. Rev. B 54, (1996) R3710. 


\section{Figure Captions}

Fig. 1 Temperature dependencies of the magnetization of $\mathrm{HoFe}_{4} \mathrm{Ge}_{2}$ measured in a $1 \mathrm{kOe}$ applied magnetic field upon ZFC, FCC and FCW conditions. The inset is the expanded view of the details of the regions around $T_{N}$ and $T_{f 1}$.

Fig. 2 Temperature dependencies of the magnetization of $\mathrm{DyFe}_{4} \mathrm{Ge}_{2}$ measured in 5, 10, 15 and $25 \mathrm{kOe}$ applied magnetic fields upon ZFC, FCC, and FCW conditions and 30 and $50 \mathrm{kOe}$ applied magnetic field upon ZFC and FCC conditions. The inset of (c) shows the expanded area near $T_{f 1}$. The inset of (e) shows the field dependence of the temperature of $T_{f 2}$.

Fig. 3 Temperature dependencies of the real $\chi$ ' (a) and imaginary component $\chi$ ” (b) of the ac susceptibility of $\mathrm{HoFe}_{4} \mathrm{Ge}_{2}$ collected in a 5Oe ac field, zero dc field and frequencies from 1 to $1000 \mathrm{~Hz}$.

Fig. 4 Magnetization isotherms of $\mathrm{HoFe}_{4} \mathrm{Ge}_{2}$ measured in applied magnetic fields from -140 to $140 \mathrm{kOe}$ at $5 \mathrm{~K}$ (a), 0 to $140 \mathrm{kOe}$ from 10 to $60 \mathrm{~K}$ (b), and derivative of magnetization with respect to the applied magnetic field from 5 to $60 \mathrm{~K}$ (c). A weak ferromagnetic signature is seen in all $M(H)$ data because of the minor ferromagnetic $\mathrm{Fe}$ impurity. 
Fig. 5 Isothermal magnetization curves at 2, 5, 10 and $15 \mathrm{~K}$ measured twice: first time after zero field cooling of thermally demagnetized sample, second time immediately following the first cycle. A weak ferromagnetic signature is seen in all $M(H)$ data because of the minor ferromagnetic Fe impurity.

Fig. 6 Time dependence of the isothermal remanent magnetization at $5 \mathrm{~K}$. The solid line represents the fit to the curve.

Fig. 7 Temperature dependencies of the heat capacity of $\mathrm{HoFe}_{4} \mathrm{Ge}_{2}$ measured in magnetic fields ranging from 0 to $50 \mathrm{kOe}$. The insets (a) and (b) show the expanded view of the details in the regions around $T_{f 2}$ and $T_{f 1}$, respectively.

Fig. 8 Temperature dependencies of the electrical resistivity of $\mathrm{HoFe}_{4} \mathrm{Ge}_{2}$ upon heating and cooling between 2 and $100 \mathrm{~K}$ in $0,1,10,20$ and $30 \mathrm{kOe}$ magnetic fields (a-e). Temperature dependence of the first derivative of the electrical resistivity with respect to temperature $(d \rho / d T)$ upon heating under zero magnetic field (f).

Fig. 9 Temperature dependence of the magnetoresistance of $\mathrm{HoFe}_{4} \mathrm{Ge}_{2}$ at $30 \mathrm{kOe}$ from 2 to $60 \mathrm{~K}$ (a). Magnetic field dependence of the magnetoresistance of $\mathrm{HoFe}_{4} \mathrm{Ge}_{2}$ from 2 to $50 \mathrm{~K}$ in both magnetization (solid symbol) and demagnetization paths (open symbol) (b). 


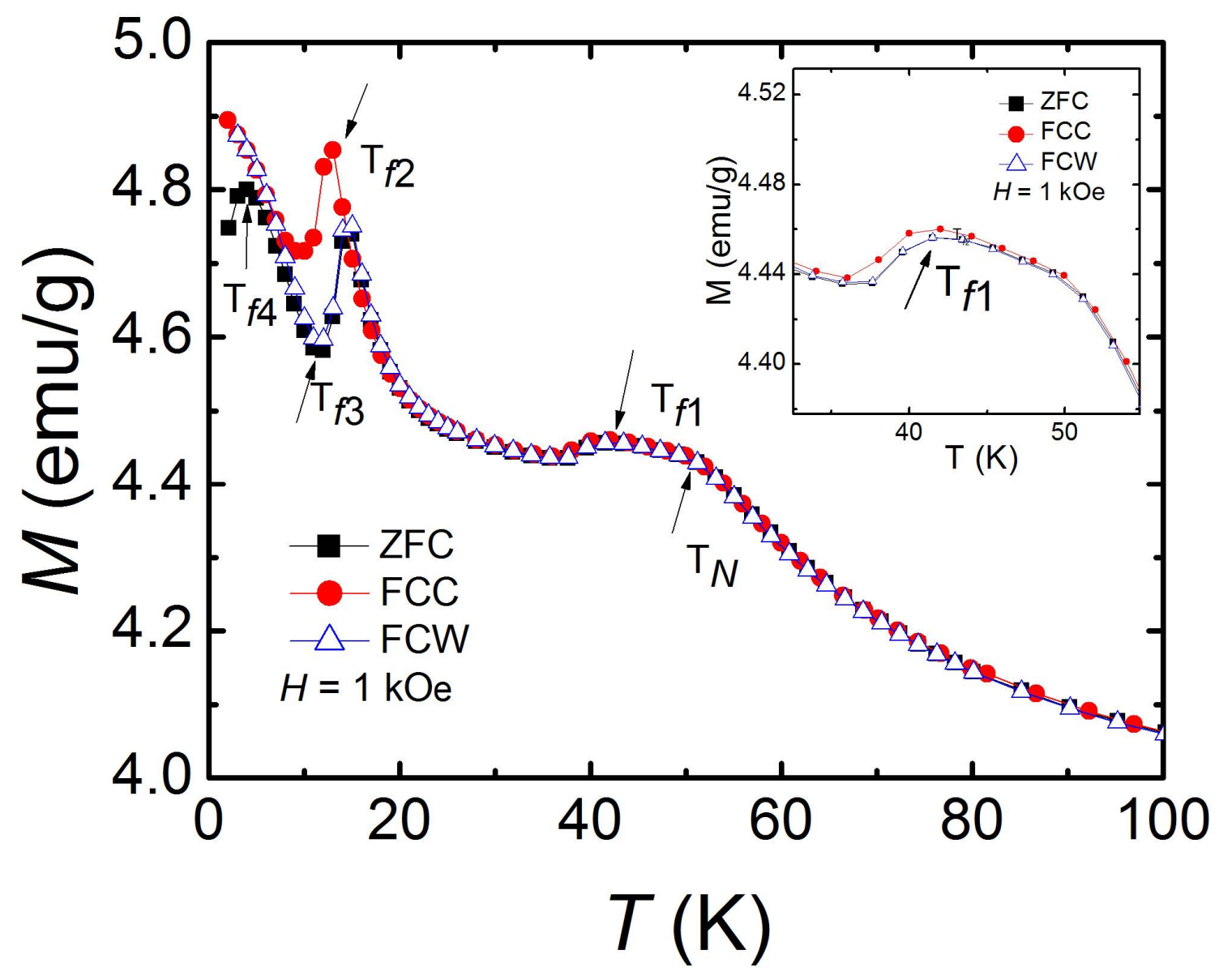

Fig. 1 Temperature dependencies of the magnetization of $\mathrm{HoFe}_{4} \mathrm{Ge}_{2}$ measured in a $1 \mathrm{kOe}$ applied magnetic field upon ZFC, FCC and FCW conditions. The inset is the expanded view of the details of the regions around $T_{N}$ and $T_{f 1}$. 

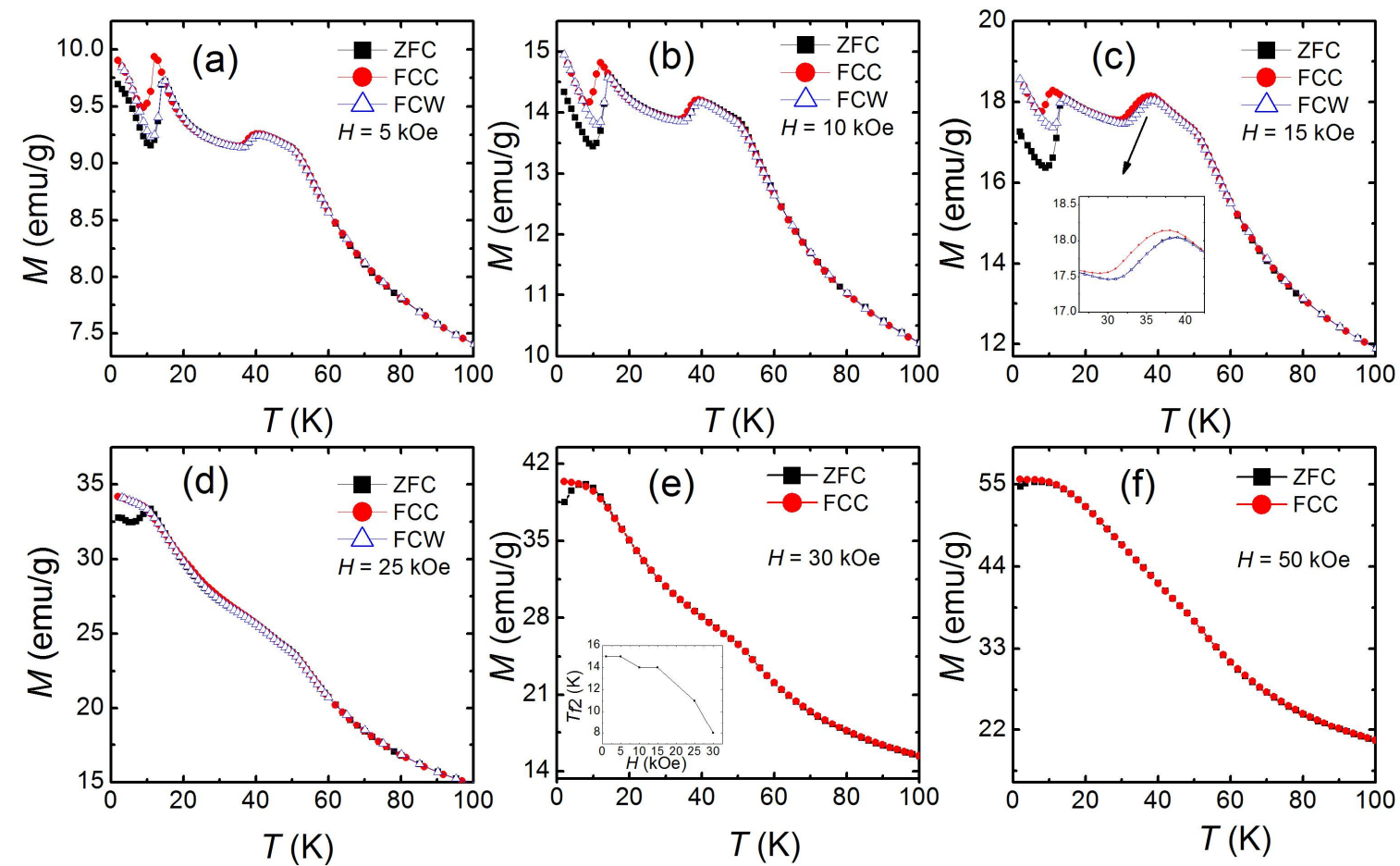

Fig. 2 Temperature dependencies of the magnetization of $\mathrm{DyFe}_{4} \mathrm{Ge}_{2}$ measured in 5, 10, 15 and $25 \mathrm{kOe}$ applied magnetic fields upon ZFC, FCC, and FCW conditions and 30 and 50 kOe applied magnetic field upon ZFC and FCC conditions. The inset in (c) shows the expanded area near $T_{f 1}$. The inset in (e) shows the field dependence of $T_{f 2}$. 

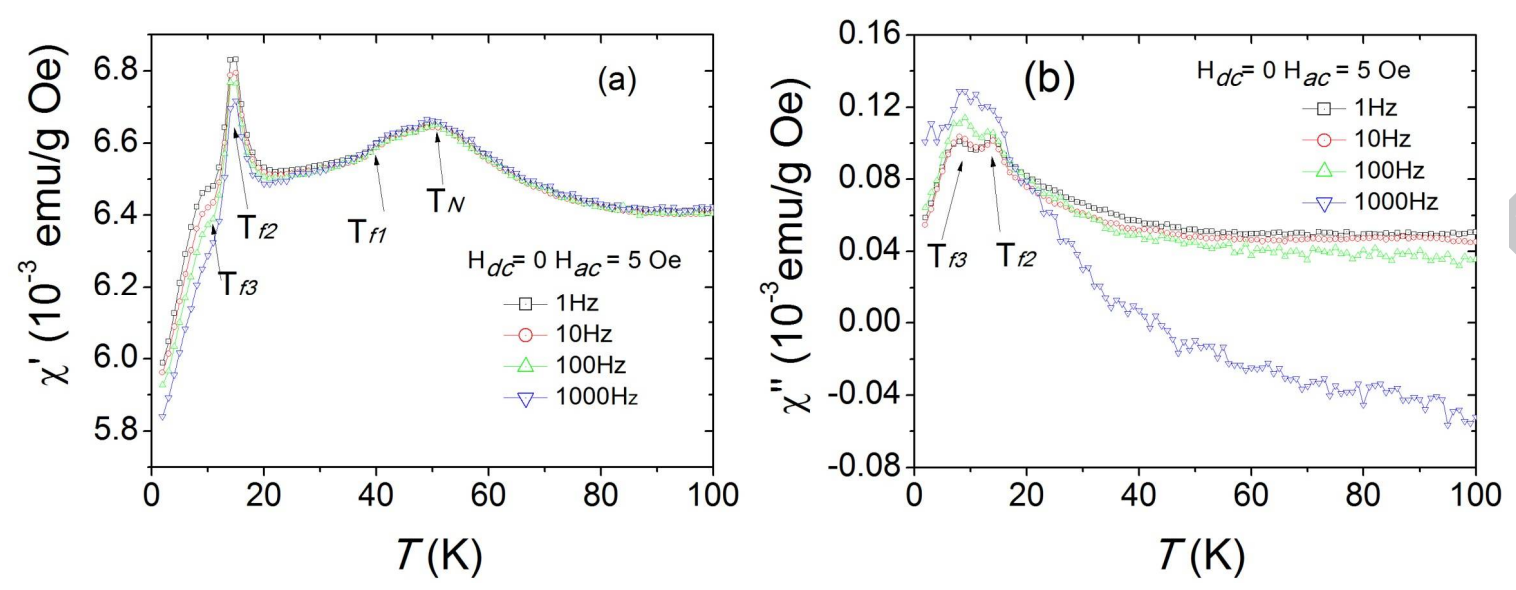

Fig. 3 Temperature dependencies of the real $\chi^{\prime}$ (a) and imaginary component $\chi^{\prime \prime}$ (b) of the ac susceptibility of $\mathrm{HoFe}_{4} \mathrm{Ge}_{2}$ collected in a 5Oe ac field, zero dc field and frequencies from 1 to $1000 \mathrm{~Hz}$. 

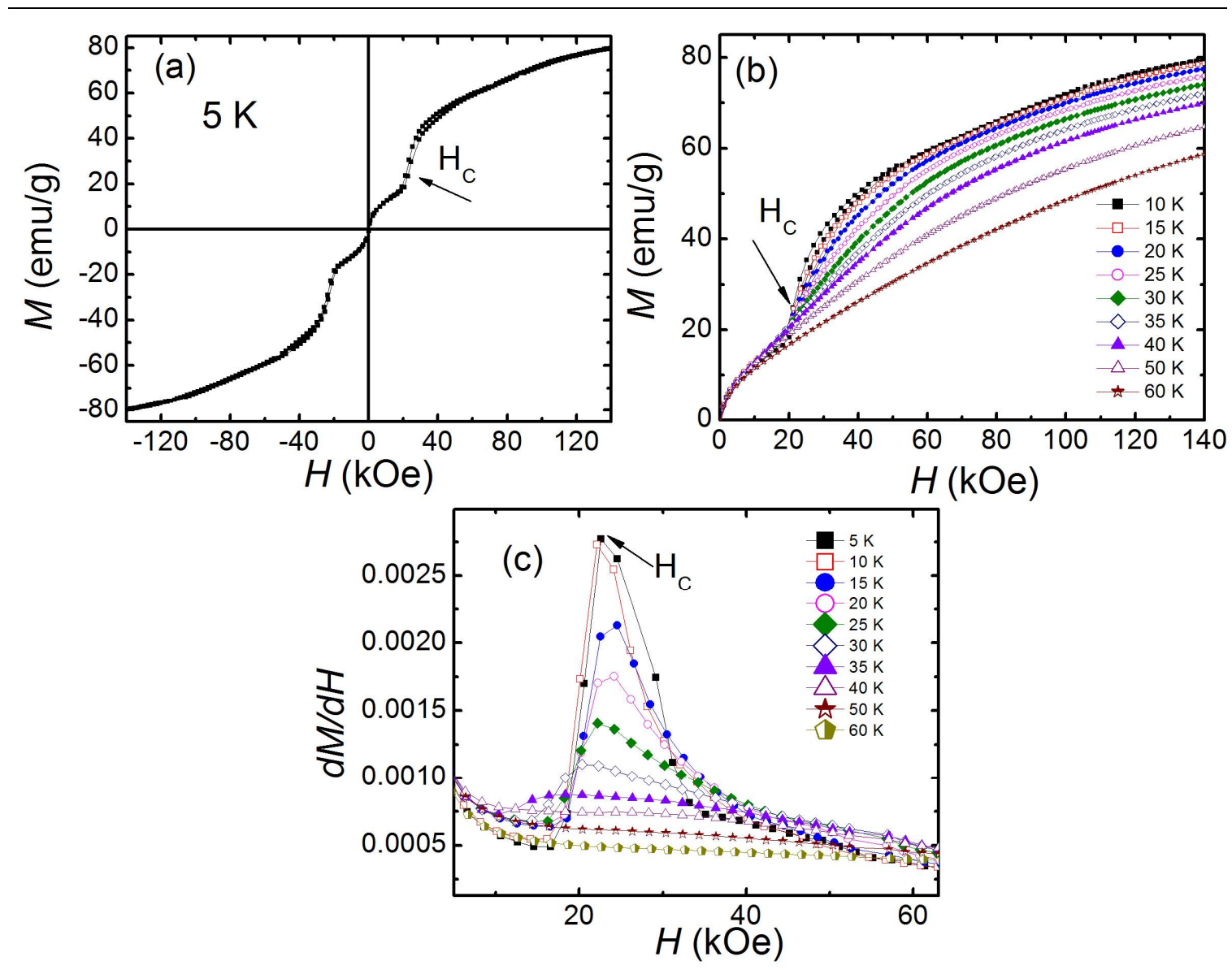

Fig. 4 Magnetization isotherms of $\mathrm{HoFe}_{4} \mathrm{Ge}_{2}$ measured in applied magnetic fields from -140 to $140 \mathrm{kOe}$ at $5 \mathrm{~K}$ (a), 0 to $140 \mathrm{kOe}$ from 10 to $60 \mathrm{~K}$ (b) and derivative of magnetization with respect to the applied magnetic field from 5 to $60 \mathrm{~K}$ (c). A weak ferromagnetic signature is seen in all $M(H)$ data because of the ferromagnetic $\mathrm{Fe}$ impurity. 

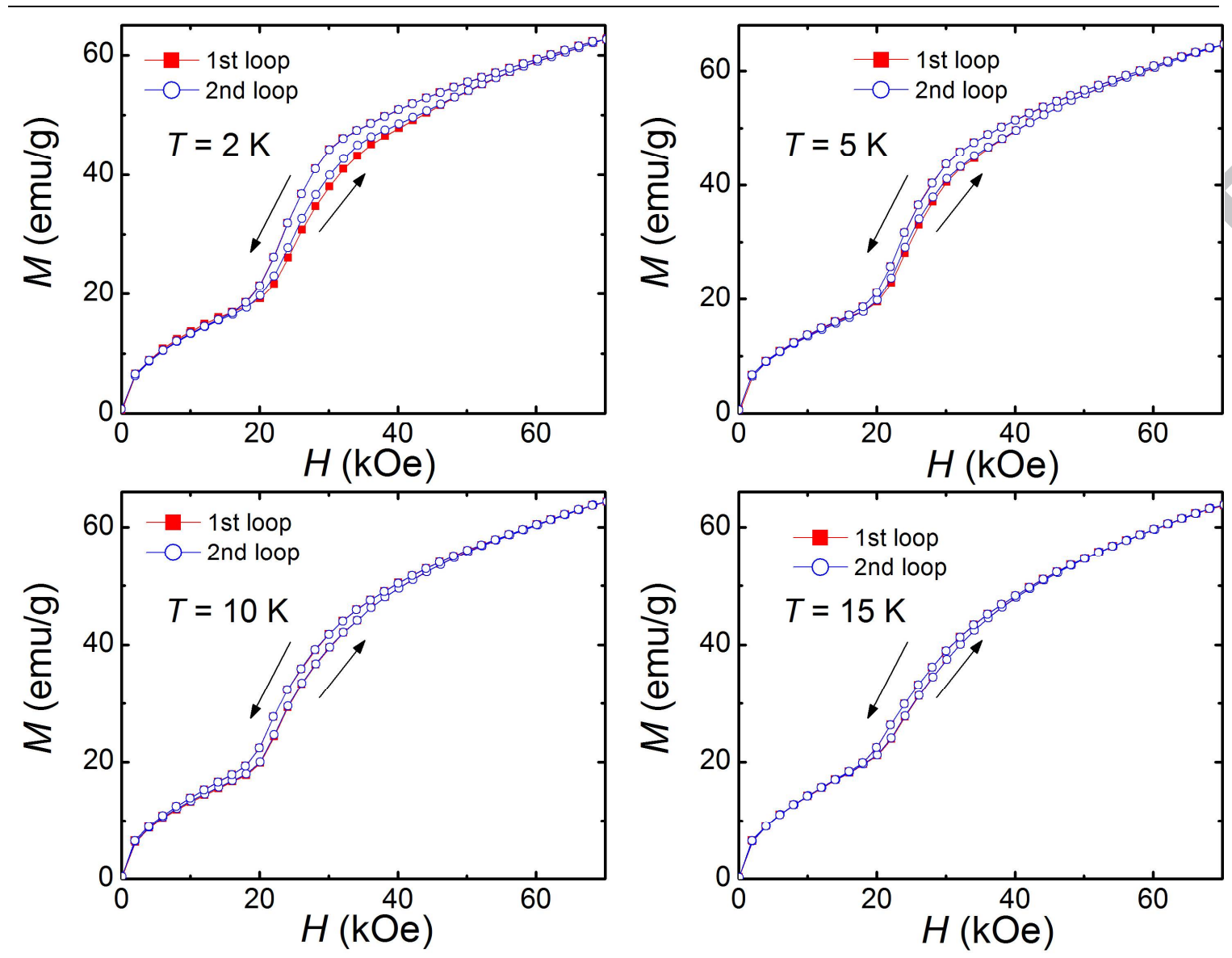

Fig. 5 Isothermal magnetization curves at 2, 5, 10 and $15 \mathrm{~K}$ measured twice: first time after zero field cooling of thermally demagnetized sample, second time immediately following the first cycle. A weak ferromagnetic signature is seen in all $M(H)$ data because of the minor ferromagnetic Fe impurity. 


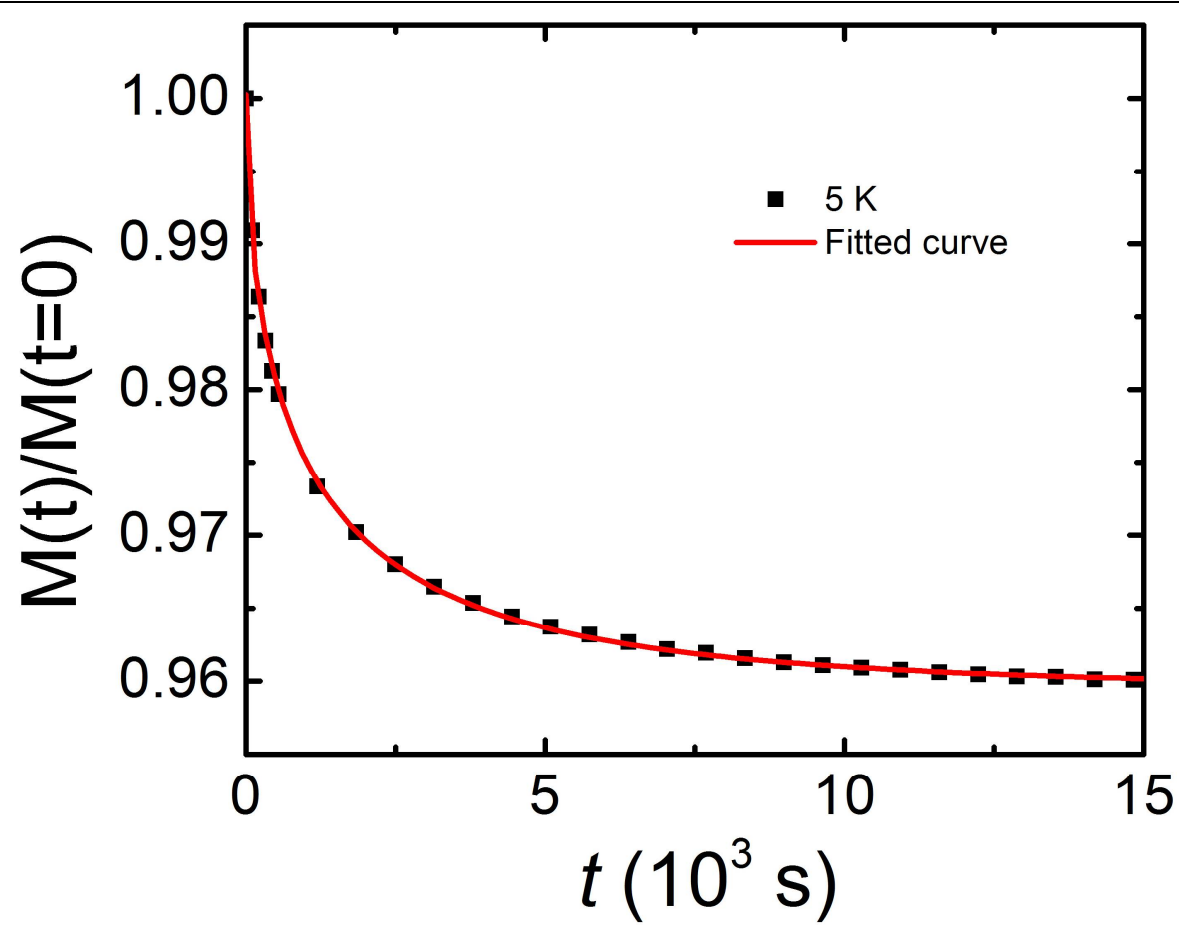

Fig. 6 Time dependence of the isothermal remanent magnetization at $5 \mathrm{~K}$. The solid line represents the exponential fit to the curve. 


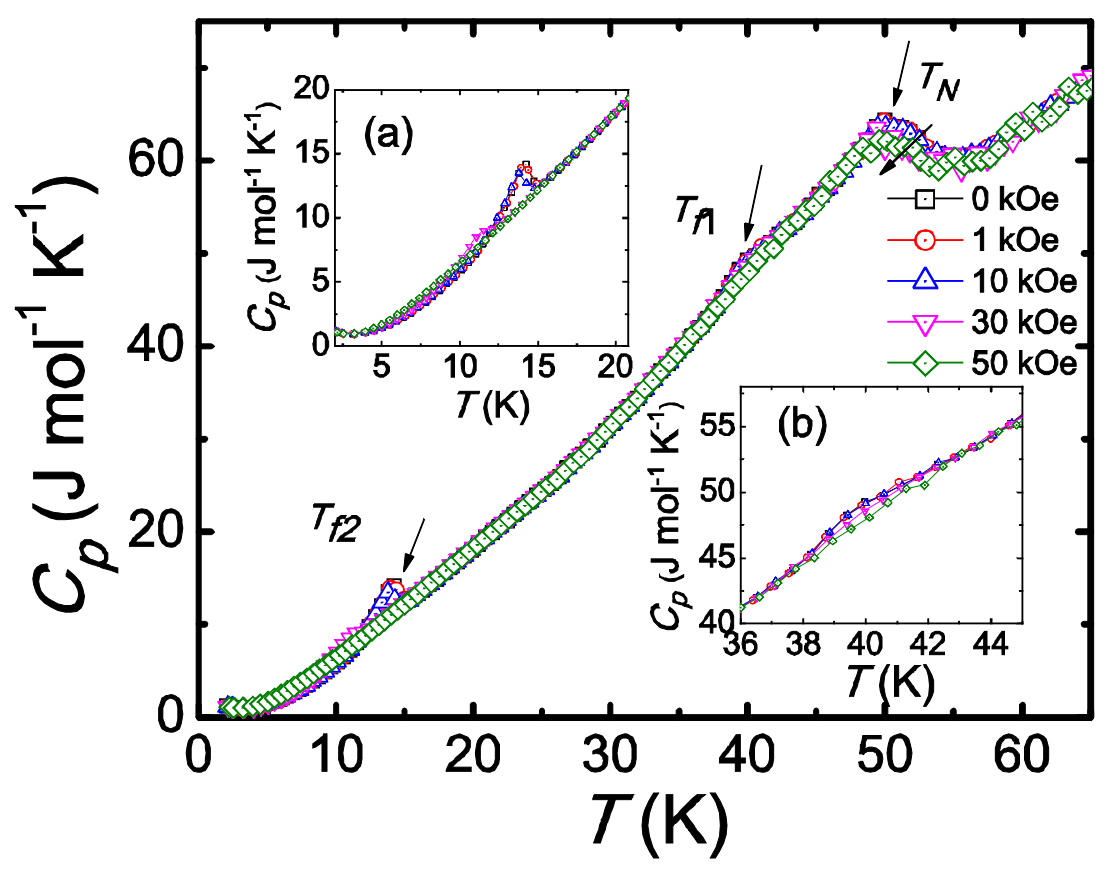

Fig. 7 Temperature dependencies of the heat capacity of $\mathrm{HoFe}_{4} \mathrm{Ge}_{2}$ measured in magnetic fields ranging from 0 to $50 \mathrm{kOe}$. The insets (a) and (b) show the expanded view of the details in the regions around $T_{f 2}$ and $T_{f 1}$, respectively. 

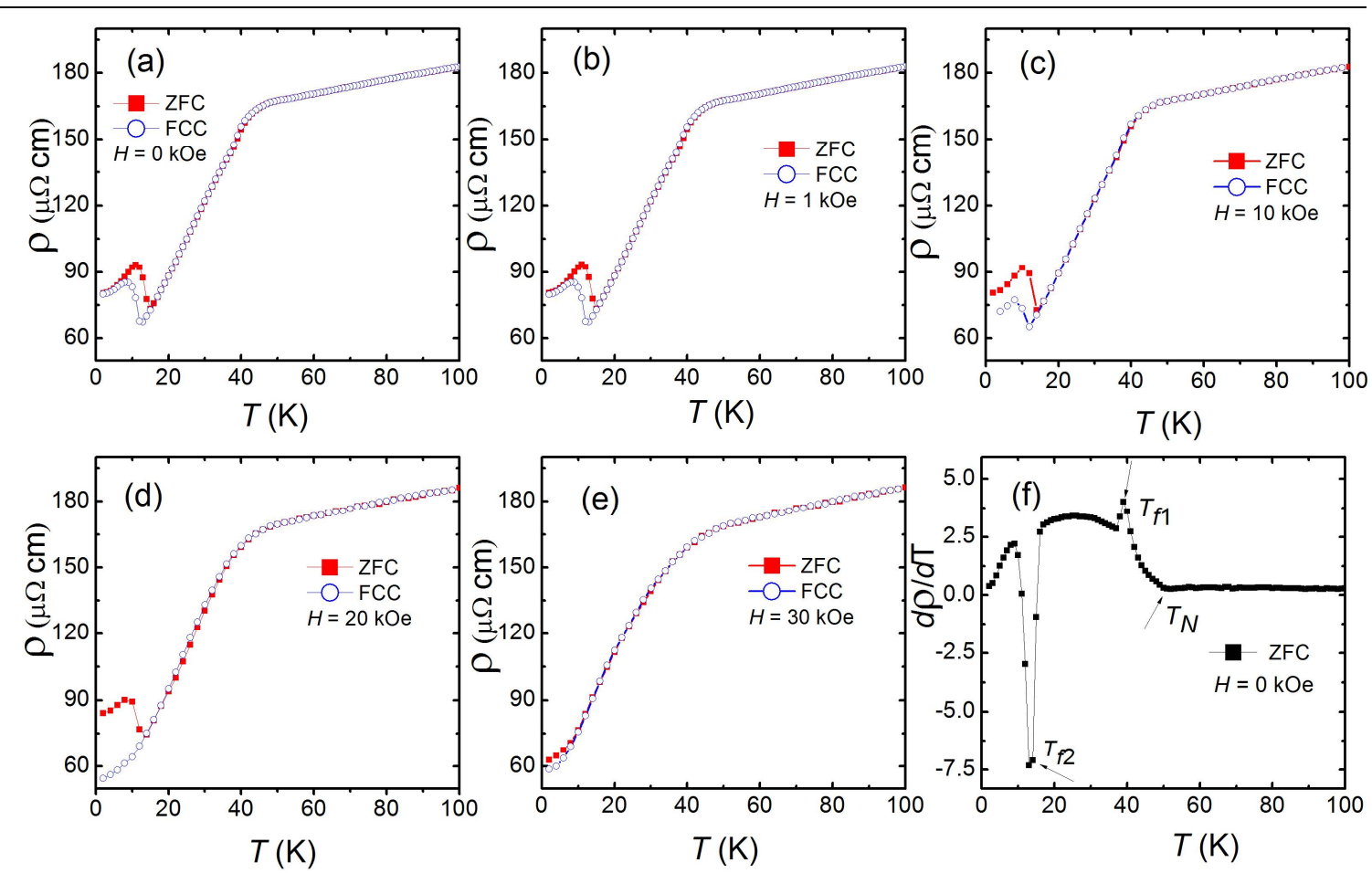

Fig. 8 Temperature dependencies of the electrical resistivity of $\mathrm{HoFe}_{4} \mathrm{Ge}_{2}$ measured upon heating and cooling between 2 and $100 \mathrm{~K}$ in $0,1,10,20$ and $30 \mathrm{kOe}$ magnetic fields ( $\mathrm{a}$ e). Temperature dependence of the first derivative of the electrical resistivity with respect to temperature $(d \rho / d T)$ upon heating in a zero magnetic field (f). 

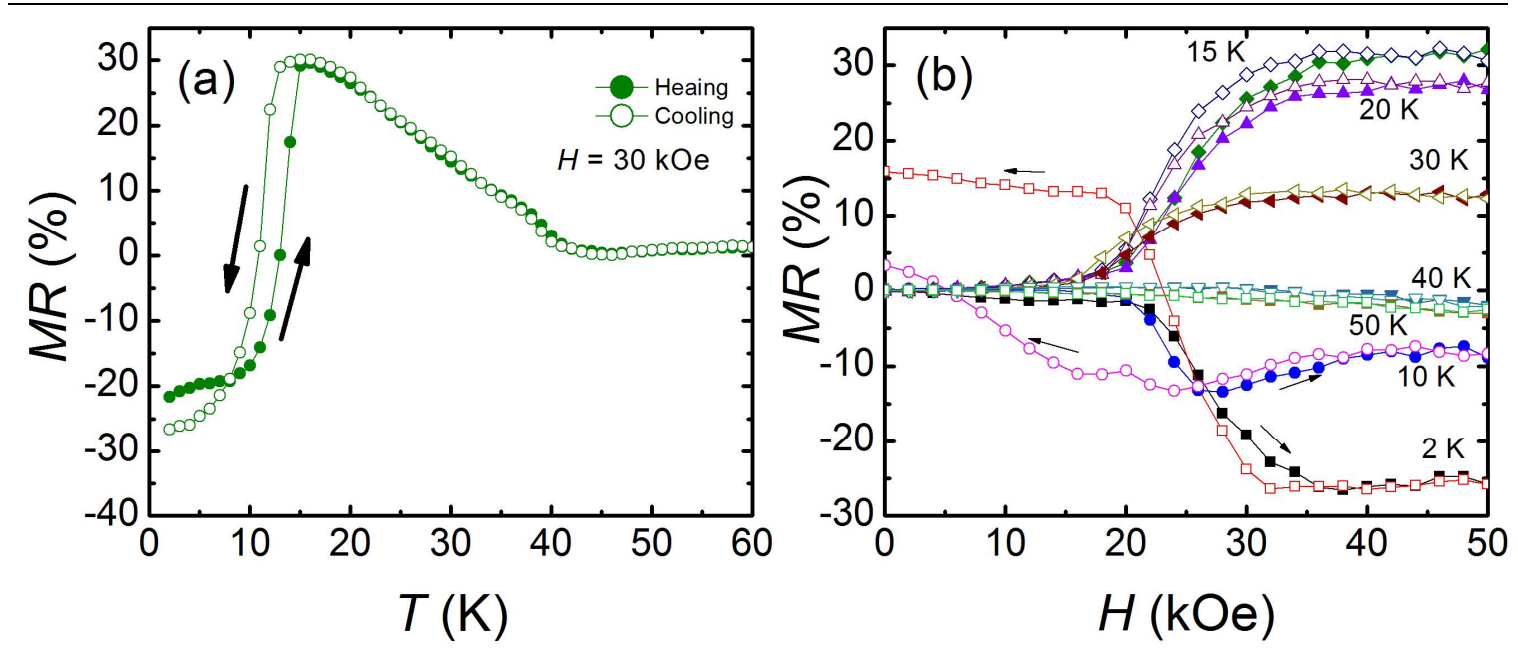

Fig. 9 Temperature dependence of the magnetoresistance of $\mathrm{HoFe}_{4} \mathrm{Ge}_{2}$ at $30 \mathrm{kOe}$ from 2 to $60 \mathrm{~K}$ (a). Magnetic field dependence of the magnetoresistance of $\mathrm{HoFe}_{4} \mathrm{Ge}_{2}$ from 2 to $50 \mathrm{~K}$ in both magnetization (solid symbols) and demagnetization paths (open symbols) (b). 